\title{
Circuit
}

Musiques contemporaines

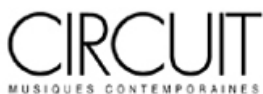

\section{Table ronde autour de Pousseur. Table ronde animée par Michel Gonneville, le 23 janvier 2001, sur l'oeuvre d'Henri Pousseur, autour de quatre de ses textes : " Mort de Dieu et crise de l'art ", " La musique ici aujourd'hui ", " Le sacré et la musique aujourd'hui? " et " Petit bilan d'une recherche aussi têtue que sinueuse " Roundtable}

Volume 12, numéro 1, 2001

Henri Pousseur : visages

URI : https://id.erudit.org/iderudit/902238ar

DOI : https://doi.org/10.7202/902238ar

Aller au sommaire du numéro

Éditeur(s)

Les Presses de l'Université de Montréal

ISSN

1183-1693 (imprimé)

1488-9692 (numérique)

Découvrir la revue

Citer ce document

(2001). Table ronde autour de Pousseur. Table ronde animée par Michel Gonneville, le 23 janvier 2001, sur l'oeuvre d'Henri Pousseur, autour de quatre de ses textes : " Mort de Dieu et crise de l'art ", " La musique ici aujourd'hui ", "Le sacré et la musique aujourd'hui? » et « Petit bilan d'une recherche aussi têtue que sinueuse ». Circuit, 12(1), 29-42. https://doi.org/10.7202/902238ar
Résumé de l'article

Retranscription de la table ronde subséquente, où les participants, Isabelle Panneton, compositrice, Jean Boivin, musicologue, et Michel Robert, professeur de philosophie, interrogent Henri Pousseur à partir de leur lecture de quatre textes de celui-ci. En relation avec ces textes, les sujets abordés touchent aussi bien la fonction sociale actuelle de la musique et l'éducation musicale que... le bonheur. 


\title{
Table ronde autour de Pousseur
}

\author{
Table ronde animée par Michel Gonneville, le 23 jan- \\ vier 2001, sur l'œuvre d'Henri Pousseur, autour de \\ quatre de ses textes : "Mort de Dieu et crise de \\ l'art ", "La musique ici aujourd'hui ", " Le sacré et la \\ musique aujourd'hui ? " et "Petit bilan d'une \\ recherche aussi têtue que sinueuse ".
}

Participants : Henri Pousseur, Isabelle Panneton (compositrice et professeur de composition et de techniques d'écriture à l'Université de Montréall, Michel Robert (professeur de philosophie au cégep du Vieux-Montréal, mélomane; intéressé par toutes les questions d'esthétique) et Jean Boivin (musicologue, professeur à l'école de musique de l'Université de Sherbrooke, auteur d'un livre sur la classe de Messiaen, paru aux éditions Bourgois).

Michel Gonneville. - Devant un homme à l'esprit aussi riche qu'Henri Pousseur, il est très difficile de se limiter dans le temps. En conséquence, et afin de concentrer le questionnement auquel nous soumettrons Henri Pousseur, mes trois invités, Isabelle Panneton, Michel Robert et Jean Boivin lui poseront quelques questions, lui feront part des commentaires qui leur sont venus à la lecture des textes que je leur ai fournis, c'est-à-dire trois textes assez denses d'Henri Pousseur, et même un quatrième, beaucoup plus récent, dans lesquels Henri Pousseur développe certaines idées parfois philosophiques sur la fonction sociale de la musique.

Le premier texte, "Mort de Dieu et crise de l'art», est tiré d'un livre paru en 1972. Musique sémantique société. Cet article a été rédigé en relation avec un exposé présenté au cours de l'été de 1968, au couvent des dominicains de la Sarthe, dans la vallée de la Meuse (belge), devant un auditoire entièrement théologique. Je cite Henri Pousseur :

Soucieux de recyclage, mes hôtes s'étaient penchés sur le paradoxe d'une théologie de la mort de Dieu et m'avaient prié de les entretenir des faits artistiques susceptibles d'illustrer ou d'éclairer ce problème. 
Dans ce texte, on se penche sur certains aspects de la philosophie esthétique, comme l'idéalisme et le matérialisme, où, par exemple, respect excessif de la tradition et volonté exacerbée de rupture sont renvoyés dos-à-dos, ou mieux, si vous voulez, sont invités à se réconcilier, à se dépasser dans le cadre de nouvelles propositions esthétiques où l'esprit du philosophe allemand Ernst Bloch vient s'insérer tout à fait naturellement.

Le deuxième texte s'intitule "La musique ici aujourd'hui", publié en trois livraisons, en 1977, dans la revue Socialisme.

Dans cet article, partant de considérations générales sur l'utilité de la musique, donc sur sa fonction, Pousseur parcourra I'histoire en cherchant à voir comment cette activité humaine s'y inscrit. II cherche ainsi à distinguer, parmi les fonctions que la musique a pu prendre, celles qui pourraient être moins reluisantes, à cause de leur collusion avec les mécanismes d'oppression, de celles qui sont véritablement porteuses d'avenir, de libération et d'accomplissement, jusqu'à aborder notre époque où "art de masse" et "art savant" coexistent et s'interpellent, où "conformisme" et "esprit de rupture " s'opposent, où l'urgence d'une pensée critique se fait plus que jamais sentir, où la pratique musicale comme l'enseignement devrait refléter ce qu'une telle conscience a pu appréhender.

Le troisième texte s'intitule "Le sacré et la musique aujourd'hui?", publié en 1982 dans la revue Corps écrits.

L'auteur y avove son allergie envers une certaine conception du sacré qui lui apparaît étroitement associée aux phénomènes d'abus de pouvoir et d'oppression et contre laquelle il lui semble urgent de continuer à lutter. Simultanément pourtant, quelque chose cache, obstrue cette notion même de sacré et continue ainsi sourdement à interpeller Pousseur, à tâcher de le séduire, à lui promettre des lumières sans égales. Le compositeur, dans ce texte, va s'efforcer de clarifier le plus honnêtement possible les liens, même cachés, qu'il peut y avoir entre sa démarche de compositeur et les différentes couches de conscience dans lesquelles résonne cette notion de sacré, depuis une inflexion autant que possible lucidement articulée jusqu'aux atavismes secrets toujours à l'œuvre.

II s'agit donc de textes très riches auxquels j'ai ajouté un quatrième texte de référence, paru en 1998, aux Presses de l'Université de Louvain, dans la collection d'écrits de l'institut Orpheus, qui s'appelle: "Petit bilan d'une recherche aussi têtue que sinueuse", dont le titre dit assez bien ce dont il s'agit et dont l'intérêt réside dans sa date tout à fait récente et dans ce qu'elle relie cette fois les formulations plus générales et abstraites des trois textes précédents aux actions bien concrètes que Pousseur a pu mener tout au long de sa carrière de compositeur, de pédagogue et de théoricien.

Isabelle Panneton. - J'aimerais d'abord souligner qu'à l'époque où j'ai lu un premier ouvrage d'Henri Pousseur Musique sémantique société, au début des 
années 80 , je plongeais également dans certaines œuvres de Roland Barthes et, à la relecture des textes que j'ai reçus de Michel Gonneville, I'un a convoqué l'autre, ce qui explique un peu les citations que j'ai choisies. Du côté d'Henri Pousseur, la lecture des textes a été d'autant plus importante qu'elle a accompagné, dans mon cheminement, la découverte de la musique du $x x^{e}$ siècle. J'ai rouvert ce livre et j'ai découvert qu'il était crayonné d'un bout à l'autre; i'ai réalisé à quel point ses textes avaient été déterminants dans la compréhension que j'ai eve non seulement de la musique du passé sur laquelle Henri Pousseur a jeté un regard d'une très grande lucidité, mais aussi de l'œuvre de Webern dont il parle avec une pertinence tout à fait remarquable. En me préparant pour cette intervention, i'ai hésité entre deux options, deux possibilités de questions; I'une du côté de tout ce que soulève Henri Pousseur lorsqu'il explique les motifs de son refus du tabula rasa qui s'opérait au cours des années 50 et 60 à travers l'œuvre de ses collègues; l'autre, du côté d'un projet de société dont il parle généreusement et qui occupe une place importante, je crois, dans sa réflexion. J'ai opté pour cette deuxième option et je commencerais, avant d'arriver à des questions à Henri Pousseur, par lancer quelques idées dont certaines sont peutêtre des lieux communs, mais nécessaires, je crois.

Je commencerai par quelques réflexions sur l'écoute qui est d'abord, à mon avis, un processus d'intériorisation. C'est vrai mécaniquement puisque l'oreille agit comme un entonnoir qui reçoit les sons et les fait converger vers l'oreille interne, mais c'est vrai aussi à un niveau beaucoup plus important puisque le cerveau décode, il associe une signification au bruit, il s'approprie l'espace et il développe, par exemple, un sens de la temporalité à travers le discours musical. L'écoute est donc un processus intime. Le compositeur qui écrit est à la fois à l'écoute de son époque et de lui-même. Il est à la merci d'une part d'obscur, d'une part d'indéchiffrable en lui-même, puisque l'inconscient est en travail dans toute œuvre de création. L'auditeur qui écoute fait éventuellement face à son époque, on le souhaite, et à ce que Roland Barthes nomme la "signifiance" qu'il définit ainsi :

Ce qui est écouté ici et là, principalement dans le chant de l'art dont la fonction est souvent utopiste, ce n'est pas la venue d'un signifié, objet d'une reconnaissance ou d'un déchiffrement, c'est la perception même, le miroitement des signifiants sans cesse remis dans la course d'une écoute qui en produit sans cesse de nouveaux, sans jamais arrêter le sens'. Ce phénomène de miroitement s'appelle la signifiance.

1. C'est Isabelle Panneton qui souligne.

J'insiste sur "sans jamais arrêter le sens » parce que je crois que de tous les arts la musique est le plus mouvant, non seulement parce qu'il se déploie dans le temps, bien sûr, mais aussi parce qu'il se laisse interpréter, dans tous les sens du terme, facilement. À partir de là, je veux formuler deux réflexions : premièrement, je pense que l'écoute de la musique dite de concert ou savante est devenue aujourd'hui aseptisée. En effet, d'une part, à travers les récents développements de la technologie, le public a perdu contact avec ce qui est à l'origine du son, les instruments et très souvent aussi avec ceux qui le produisent, les interprètes. D'autre 
part, les disques, la radio et l'Internet permettent non seulement une audition, mais des auditions répétées des mêmes œuvres. On peut ainsi se renfermer dans le confort des trajectoires connues, dans le désir de retrouver des émotions ou simplement dans celui de rêvasser. Si la musique populaire a préservé un lien avec le corps des instruments et avec celui des interprètes, comme en parle très bien Henri Pousseur, d'ailleurs, dans "Musique ici et aujourd'hui ", il reste qu'il se joue très peu de musique dans les maisons ou à l'école. Les enfants ont rarement de vrais contacts avec les instruments, avec ce qui leur permettrait tant de développer une sensibilité au langage musical, que de participer, de coordonner les sons, de les organiser, de conduire un discours, de le vivre dans leur corps. Autrefois, pour avoir accès à la musique, il fallait aller au concert ou alors en jouer.

II me semble que ces réalités ont grandement contribué à accentuer, je ne dis pas à créer, mais bien à accentuer le fossé entre le public et les compositeurs. L'écoute, celle qui en principe relie deux pensées, celle qui permet de structurer une relation, est coupée de l'origine du son, dématérialisation des acteurs qui va de pair avec une sorte de schizophrénie, un état d'enfermement dans lequel peut se maintenir l'écoute aujourd'hui, indéfiniment, par l'intermédiaire des écouteurs, des haut-parleurs, etc.

Deuxièmement, j'ai parlé précédemment de l'intimité de l'écoute. II me semble qu'il s'est développé, avec l'avènement des technologies, une certaine sauvagerie. Aujourd'hui, ce qui sert à la fois de relais et d'écran entre les musiciens et les auditeurs, ce sont des techniciens du son et des appareils qui déchargent toutes sortes de musiques dans l'espace public, que ce soit dans la salle d'attente chez le médecin, dans le métro, ou au supermarché. Bref, des lieux où l'individu n'a aucun contrôle et doit encaisser, absorber passivement tout et rien. Bref, on trouve le même phénomène sauvage du côté des responsables de la programmation des stations de radio et de télévision, qui non seulement choisissent des musiques en fonction des lois, que l'on connaît bien, du marché, de la cote d'écoute, de la rentabilité, mais décident aussi à quelle musique ils vont accorder le plus de visibilité.

La création musicale contemporaine, dans la mouvance de ses miroitements, avec son caractère déstabilisateur, dans la force vitale de son statut d'art, par opposition bien sûr au produit commercial qu'on voudrait qu'elle soit, la musique, donc, peut-elle encore rejoindre un large public? J'ouvrirais ici une petite parenthèse, toute personnelle et qui est à l'origine de certaines de mes réflexions : je vis toujours très mal le moment d'une création d'une de mes œuvres, pas seulement parce que, comme bien d'autres, le doute m'assaille à propos de ce que j'ai écrit, mais aussi parce qu'au moment où je livre une œuvre au public, je renonce à une certaine forme d'intimité, cette part d'obscur, que j'ai eue avec cette œuvre. Par ailleurs, il est aussi demandé au public de renoncer à son intimité parce qu'il doit prêter l'oreille à une trajectoire ou à un sentier qu'il n'a jamais fréquenté auparavant, à un univers avec lequel il n'est pas familier. Ce sont des situations exigeantes pour les deux parties. Alors jusqu'où peuton aller dans notre démarche 
de sensibilisation à la musique d'aujourd'hui lorsque l'on connaît la multiplicité des tendances que cette expression "musique d'aujourd'hui" recouvre? Comment donner accès à notre musique sans faire le jeu de cette sauvagerie que je viens de décrire? Je cite une dernière fois Roland Barthes:

Aucune loi ne peut obliger le sujet à prendre son plaisir là où il ne veut pas aller quelles que soient les raisons de sa résistance. Aucune loi n'est en mesure de contraindre notre écoute. La liberté d'écoute est aussi nécessaire que la liberté de parole. C'est pourquoi cette notion, apparemment modeste ll'écoute ne figure pas dans les encyclopédies passées, elle n'appartient à aucune discipline reconnuel, donc l'écoute est finalement comme un petit théâtre où s'affrontent ces deux déités modernes, l'une mauvaise, l'autre bonne, le pouvoir et le désir. Plus que jamais nous voilà aux prises avec l'un et l'autre, avec la tension qui se joue entre les deux.

L'article d'Henri Pousseur qui s'intitule : "La musique ici aujourd'hui " contient un projet de "jonction libératrice entre des courants oppositionnels». Vous parlez, Henri Pousseur, de la liberté et vous rejoignez, je crois, Roland Barthes dans ce que je viens de citer. Vous parlez de la liberté avec laquelle il faut franchir l'épaisseur de ce qui sépare les musiciens populaires, les musiques populaires et marginales de la musique des musiciens. Vous mentionnez également la nécessité de la contamination et de l'enrichissement réciproque qui pourrait découler d'une telle rencontre. C'est un projet utopiste, mais, pour cela, peutêtre valable! C'est un projet qui, tout en ouvrant la voie à une diffusion plus large de la musique, comme vous le formuliez, échappe à toute préoccupation de rentabilité. C'est pour cette raison que j'ai beaucoup aimé ce passage de votre texte, la façon dont vous en parlez. Étant donné que votre article a été écrit en 1977, comment reparleriezvous aujourd'hui de ce projet? Est-ce qu'il vous paraît toujours réalisable? Est-ce que vous l'assumez toujours pleinement?

Henri Pousseur. - Oui, d'ailleurs la manière dont les musiciens m'ont accueilli tout à l'heure ${ }^{2}$, c'est un exemple... Parce que Michel Massot, ce vieux complice et ami, qui a beaucoup joué de ma musique, mais surtout qui a enseigné au Conservatoire que j'ai essayé d'animer par ma direction, est un musicien justement qui appartient à ce qu'on a appelé le troisième courant, c'est-à-dire un courant qui associe la musique venant du jazz et la musique de recherche, la musique dite "savante". Michel Massot est quelqu'un qui, par exemple, dans les nombreuses représentations publiques de la classe que l'on a appelée ici "classe de musique contemporaine", dirige en particulier des activités qu'il appelle le "rock de chambre " En fait, c'est une classe de musique de chambre, mais qui a pris des dimensions tout à fait inusitées, évidemment, et qui risque d'ailleurs, du fait des autorités qui essaient toujours de remettre les choses en ordre, d'être réprimée... Moi, je l'ai propulsée à l'époque, j'ai essayé de la protéger, mais maintenant que je ne suis plus là, il semble qu'il y ait des dangers de mauvaises intentions de la part des autorités administratives dont elle dépend. Nous sommes toujours dans une situation de résistance et de lutte. Pour en revenir à la fanfare d'ouverture, ce
2. Michel Massot avait préparé, avec une soixantaine d'élèves du Conservatoire et de la Faculté de musique de l'Université de Montréal, une grande fanfare d'accueil improvisée, présentée en guise d'ouverture de l'événement dans le hall d'entrée du Conservatoire. 
n'était pas "de chambre" ni du rock, mais une musique où se rencontrent toutes sortes de choses.

Quand j'étais directeur du Conservatoire, mon fils avait une vingtaine d'années et avait quitté ma musique assez tôt pour faire sa propre musique, laquelle était, au départ, une espèce de musique rock-pop. II jouait cette musique avec quelques copains, il avait demandé qu'on lui achète une batterie, puis il s'est remis au piano et s'est rendu compte qu'il avait besoin d'étudier. Il est allé suivre des cours de jazz à Paris, et c'est à travers lui que j'ai appris à connaître beaucoup de jeunes musiciens qui ne pouvaient pas étudier au Conservatoire, non seulement parce qu'ils n'avaient pas les diplômes, mais aussi et surtout parce que ce qu'on y enseignait ne les concernait pas, ne leur apportait rien.

Alors nous avons eu l'occasion de créer un séminaire de jazz qui a existé pendant cinq ans et où une cinquantaine de jeunes musiciens ont été formés par les meilleurs jazzmen belges, un peu plus âgés qu'eux, et par des maîtres étrangers, venus des États-Unis et d'Allemagne. II y a eu donc là tout un travail qui s'est fait, un travail de croisements, de rencontres, et qui ensuite s'est propagé. Ce séminaire n'a pas continué d'exister parce que les autorités en ont décidé ainsi. À la place, elles ont créé un département de jazz au Conservatoire de Bruxelles où la structure d'ensemble était moins favorable, je crois, mais enfin où les jeunes enseignants ont continué à travailler. Ils ont créé aussi des centres de jazz dans ce qu'on appelle en Belgique les académies de musique, qui sont des écoles de niveau inférieur. À la suite de cette création, il y a eu tout un mouvement d'infiltration d'une musique différente parmi la musique dite sérieuse, aristocratique.

Je ne dis pas que grâce à cela, c'est réussi. Non, c'est loin d'être réussi, mais enfin il y a des poches de mise en commun qui se sont créées. II y a aussi tout un travail qui continue à s'effectuer avec des hauts et des bas.

À peu près à la même époque, la radio belge m'avait demandé d'écrire une œuvre d'assez grande dimension pour l'orchestre de la radio lqui n'existe plus maintenant, ayant été supprimé pour des raisons qui sont liées à toute cette évolution de sauvagerie économiquel, et on $\mathrm{m}^{\prime}$ avait dit : "il peut y avoir un soliste ou un chœur ou quelque chose comme ça". Comme on venait de créer le séminaire de jazz et que j'avais établi un contact beaucoup plus étroit avec cet univers, j'ai pensé faire une œuvre où j'essaierais d'associer ces musiques afin d'aborder un autre angle de mon projet général, qui était de rapprocher non seulement la musique ancienne et la musique contemporaine, mais aussi, la musique occidentale dans son ensemble et, si possible un peu, les musiques populaires et les musiques non occidentales. J'ai alors entrepris de composer une œuvre pour un ensemble de jazz, un petit orchestre symphonique, et pour ne pas jouer sur simplement un "vis-à-vis» qui risquait d'apparaître comme une espèce de confrontation polémique, j'ai ajouté un troisième terme, de manière à ce qu'il y ait une circulation qui s'instaure, et ce troisième terme était un groupe de synthétiseurs là 
l'époque, vers 1980 ou 1981, c'était encore des synthétiseurs principalement monodiques). II y en avait quatre. L'un des instrumentistes jouait aussi de la percussion électronisée et un autre, du piano. Une personne pilotait le tout au pupitre de mixage.

J'ai demandé à mon fils de m'assister pour toute la partie jazz parce que je ne me considérais pas suffisamment compétent dans la grammaire de cette musique, dans sa sensibilité créative. On a donc fait ensemble une grande machine en trois parties dont la première est une espèce d'océan sonore duquel émergent, petit à petit, des îles qui sont les trois univers. Le tout s'appelle d'ailleurs les Illes déchaînées. Au début, les îles sont séparées (cette partie s'appelle "Origine des îles»), et dans la deuxième partie, qui s'appelle "Duels», les îles se confrontent deux par deux. Il y en a une, par exemple, qui écrase l'autre, puis le mouvement se renverse, et ainsi de suite jusqu'à ce qu'il y ait, à la fin, une espèce de fiesta où les trois sont ensemble.

Puis on entre dans la troisième partie qui s'appelle "Autour d'lcare» (lcare 2, celui qui a découvert grâce à sa tante Mnémosyne une cire d'abeille qui ne fond pas au soleil!). Dans cette pièce, les trois ensembles sont réunis autour d'lcare, en un seul grand "pool", et sont réarticulés en catégories perpendiculaires, c'estàdire que tous les sons de nature "corde" ou "glissée " des trois ensembles forment une famille transversale, tous les sons de nature "vent" en forment une autre, et de même tous les sons de nature "percutée», etc. Ce qui était opposé au départ s'est donc réuni en une nouvelle société, une microsociété musicale, humaine. Dans cette troisième partie, il y a un texte de Michel Butor extrait de Sur le qui-vive (c'est le numéro 3) qui est "dit". Je le disais moi-même: "Un jour l'arche pénétrera de l'autre côté des cavernes, un jour..., un jour..., » etc. C'est un texte utopisant qu'il est difficile de soutenir aujourd'hui quand on voit à quel point l'utopie s'est éloignée... Enfin, cela a été fait et, comme je le disais à des amis qui me parlaient de leur découragement, il faut continuer, il faut travailler à des choses comme celleslà qui, quand même, font leur effet et des petits...

Isabelle Panneton. - Avez-vous le sentiment, à travers ces expériences-là, que vous avez repris contact avec le public? Cette volonté de renover ne se profilaitelle pas un peu à travers vos articles?

Henri Pousseur. - Je ne voudrais pas que l'on croie que, parce que je l'ai pensé moi-même, j'espérais, par exemple, que Votre Faust, allait me mettre dans une situation de communication meilleure avec le public. Mais pas du tout! Au contraire, le public (un certain public spécialisé) s'était peu à peu familiarisé avec la musique des années 50 , tandis que cette musique que je venais de faire a beaucoup heurté mes anciens amis. J'ai donc été mis au ban! Par contre, j'ai rencontré parfois d'autres publics. Le premier concert de Votre Faust, par exemple, en 1966, à Bruxelles, a connu un succès énorme auprès du public. L'exécution des Îles déchaînées, à Bruxelles et à Liège, a rassemblé des publics qui étaient le 
public de musique contemporaine et le public de jazz, et qui se sont associés dans un même enthousiasme.

En 1970, quand nous avons créé le Centre de recherches musicales de Wallonie, nous avons organisé, avec l'ensemble Musique nouvelle, une grande manifestation fondatrice qui s'appelait "Midi-minuit " qui durait effectivement douze heures), au Palais des congrès de liège. Cet événement se déroulait dans quatre salles, dans lesquelles le public pouvait se promener et qui étaient interreliées par des systèmes de microphones et de haut-parleurs. Il y avait différents groupes de musique contemporaine, de musique baroque, de jazz, de rock, un chœur d'amateur $\grave{A}$ coeur joie, une pianiste qui jouait du Haydn et du Boulez, deux chanteuses remplaçant Cathy Berberian, un musicien folkloriste, etc.

Le public pouvait se promener et pouvait acheter, dans une salle où il y avait des jeux musicaux, des tickets avec lesquels il allait demander aux opérateurs d'ouvrir ou de fermer les haut-parleurs qui amenaient des sons dans une autre salle. Un groupe de jazz, par exemple, recevait tout d'un coup une messe de Victoria dans les mollets et devait essayer de s'adapter, rythmiquement, tonalement, si possible en préservant ce qu'il avait entamé. Puis, si c'était nécessaire, il devait se résoudre à improviser. Ça donnait des résultats extraordinaires! $\grave{A}$ un certain moment de la demi-journée, on avait ce qu'on appelait les "états généraux" où tout le monde se rassemblait dans le grand hall et faisait une improvisation collective, cela à deux reprises : avant $18 \mathrm{~h}$ et $24 \mathrm{~h}$.

$\|$ y a eu deux mille personnes qui se sont promenées là-dedans. J'étais professeur de composition, à ce moment-là, ef j'habitais dans un immeuble où il y avait un concierge, un petit monsieur très gentil, pas du tout habitué à la musique de concert, et surtout pas à la musique contemporaine! J'avais invité ce concierge, il est venu, et il m'a dit: "Monsieur Pousseur, c'est l'image d'un monde nouveau! » (Rires.) Vous vous rendez compte? Voilà, ce n'est qu'une anecdote, je ne peux pas dire grand-chose de plus! Aujourd'hui, je suis beaucoup moins optimiste qu'alors...

Isabelle Panneton. - C'est ce que je voulais savoir, en fait.

Henri Pousseur. - Mais en fait, je suis calme quand même et j'essaie d'aider les jeunes à continuer à travailler, à résister et à semer.

Michel Robert. - Pourquoi êtes-vous si pessimiste maintenant? Ce qui n'est pas "musique contemporaine " ou autre musique estil seulement " déchet»?

Henri Pousseur. - Non, non, pas du tout, ce n'est pas ce que je veux dire... Je ne suis pas pessimiste parce qu'on n'écoute pas assez la musique contemporaine. Ce n'est qu'un épiphénomène, je crois... Je suis pessimiste parce que la planète est maintenant entièrement gouvernée par une économie devenue folle et que tous les aspects de la vie sont plus ou moins pourris par ça. La coupure sur le plan de l'écoute dont Isabelle a parlé, est une coupure qui existe à tous les niveaux à cause, entre autres, des médias, des grands médias, mais pas uniquement à 
cause d'eux. Les individus, par exemple, sont de plus en plus isolés; il y a de moins en moins de cohésion sociale, que ce soit celle des familles, celle des collectivités de toutes sortes... En Occident, la société a conservé une partie de la structure qu'on lui connaît depuis plusieurs siècles, mais on a détruit les structures des sociétés extra-européennes d'une manière particulièrement sauvage alors qu'elles ont des cultures qu'il faudrait essayer de préserver parce qu'elles nous apportent énormément de richesse. On les utilise, naturellement, dans la mesure où elles rapportent, mais en les édulcorant, en les détruisant quand même... C'est cela, donc, qui me rend pessimiste! On divise, on sépare, on morcelle, pour régner, certes, mais surtout pour accumuler, finalité des finalités, objectif qui finira par s'étrangler lui-même!

En 1968, qui reste pour moi une année phare, malgré les maladresses considérables qui ont été perpétrées par les acteurs de l'époque, y compris nousmêmes (cela avait commencé dans les années 50), je me rappelle avoir reçu une carte de Michel Butor, avec qui j'étais déjà très lié (je le suis toujours), dans laquelle il me disait: "Regarde comme l'appareil se paralyse, mais on dirait que quelque chose va se passer. "Et nous nous sommes vus à Paris, quelques semaines plus tard, au début de juin, et il m'a dit : "Voilà! Un certain nombre de choses ont été mises sur le tapis, mais maintenant elles sont déjà remises sous la table...» En effet, on a joué sur la peur des gens pour instaurer un régime de rétrospectivité de plus en plus généralisé. J'ai cru que cela allait durer dix ou quinze ans et puis que la vague repartirait dans l'autre sens, mais la situation perdure. Ce n'est pas à l'échelle de l'individu, c'est à l'échelle de l'Histoire, c'est-à-dire à long terme comme diraient certains historiens, tel Fernand Braudel.

Je n'ai pas idée du temps qu'il faudra pour que nous surmontions cette nouvelle difficulté. Mais je crois que nous assistons à l'instauration d'une domination totale de l'économie capitaliste. Ce phénomène que Marx avait prévu au XIX siècle, se réalise d'une manière peut-être imprévue mais cet impérialisme du capitalisme mène au profit pour lui-même devenant le seul but de l'activité humaine. Vous savez comme moi qu'il y a une masse d'argent beaucoup plus importante que celle qui sert à l'économie réelle, c'est-à-dire aux échanges et au travail. Cette masse d'argent circule uniquement pour la spéculation. C'est une espèce de cancer de la planète! Est-ce qu'on arrivera à trouver une solution à ce problème-là ? Peut-être! II y a des tentatives, des résistances qui se manifestent... Mais est-ce qu'elles auront la force de renverser ce mouvement-là ? Je ne sais pas. Voilà ce qui me rend jusqu'à un certain point, momentanément, pessimiste. Peut-être que nos enfants (ou leurs petits-enfants) auront le bonheur de connaître enfin autre chose? En général, la vie, l'humanité finissent par trouver des portes de sortie...

Michel Robert. - Mais est-ce que la musique peut continuer à être utopiste à ce niveau-là? Car c'était intéressant ce que vous disiez par rapport à l'ego ou ce que vous avez appelé calmement le cartésianisme. On peut quand même constater aujourd'hui que l'individualisme reste encore extrêmement prégnant. Est-ce que la 
musique peut encore continuer au niveau de son message utopiste à remettre en question cet ego?

Henri Pousseur. - Elle devrait! Je pense qu'elle peut agir de plusieurs manières. La musique peut avoir une action politique au sens large, sûrement pas au service de tel ou tel parti ou d'une idéologie dogmatique, mais en elle-même. D'après ses propres nécessités, elle peut avoir une action politique, et le modèle de Webern est là. Certains ont dit que Webern avait été nazi. C'est absolument aberrant! || avait un gendre nazi, certes, mais lui-même était un artiste soucieux d'éthique, de l'éthique sociale la plus exigeante. II avait dirigé un chœur d'ouvriers avec une rigueur artistique extraordinaire! Son modèle, que j'ai essayé de décrire tout à I'heure, c'est un modèle de société autre, me semble-til, où ce qui compte, ce sont les hommes, leur bonheur, au sens large du terme, leur humanité maximale.

La musique contemporaine, donc, a plusieurs façons d'agir en ce senstlà et on peut la classer en plusieurs catégories plus ou moins affirmées.

D'une part, la musique peut exprimer les crises (tout l'expressionnisme en est un bel exemple). C'est une partie qui parle des douleurs, des difficultés, des contradictions, etc. C'est ce que j'appelle le courant expressionniste, avec une partie révolutionnaire.

D'autre part, il y a la partie plus expérimentale et utopiste, c'est-à-dire qui essaie de proposer des modèles. J'ai écrit une série d'œuvres de ce genre, des œuvres dites "Ouvertes". II y a, par exemple, lcare apprenti, pièce écrite en 1970 lje me suis rappelé tout à l'heure que je l'avais probablement conçue à Montréal!). Cette œuvre est à la fois extrêmement ouverte et structurée : c'est une machine cybernétique pour un nombre indéterminé de musiciens qui doivent vraiment créer ensemble un événement musical, sur la base de ce que je leur fournis comme aide.

À partir d'un travail attentif, l'exécution de ce genre d'œuvre permet d'entendre une musique qui est au moins aussi bien formée, aussi transparente et aussi éloquente qu'une musique entièrement rédigée. II s'agit alors d'une création véritablement collective et non autoritaire, où tous les apports sont $d^{\prime}$ importance qualitative égale.

Cela s'inscrit dans une série d'œuvres que j'ai commencées dès la fin des années 1950, inspirées de l'exemple américain, mais aussi parce que la musique électroacoustique nous apprenait qu'on pouvait mélanger les choses de différentes manières, les permuter. On a commencé des œuvres ouvertes, variables (Stockhausen avait écrit son Klavierstück XI, Boulez, sa Troisième Sonate), et moi je me suis tout de suite intéressé au problème de la musique ouverte pour plusieurs musiciens. J'ai écrit Mobile pour deux pianos et, en 1960, Répons pour sept musiciens à laquelle on a ajouté un acteur et, quelques années plus tard, un texte que Michel Butor avait écrit en étudiant la structure et en jouant avec elle d'une manière remarquable. En 1970, i'ai écrit Les Éphémérides d'lcare 2, pour vingt musiciens avec un chef qui les aide plutôt qu'il ne les commande. Enfin, il n'y a pas 
longtemps, j'ai écrit une œuvre pour un orchestre de trente-six musiciens, organisé en six groupes de six. II faut des organisations cellulaires pour que les petits groupes puissent se concerter et jouer les uns avec les autres. II ne faut pas qu'il y ait de masse (enfin il peut y avoir aussi des effets de masse, c'est parfois utile), mais pour résoudre ce problème qui est celui de l'entropie, les organisations à plusieurs niveaux sont utiles l'entropie est une notion de la théorie de la communication, de la théorie de l'information, de la physique aussi, selon laquelle, lorsqu'on mélange des éléments différents, il y a une tendance à une homogénéité de plus en plus grande). Si on a deux musiciens qui sont libres, qui ont une possibilité d'inventer des choses, chacun pour soi, et qu'ils le font sans coordination, ça risque de se brouiller. S'il y en a trois, quatre, cinq, six, sept, ça se brouille de plus en plus et on obtient une soupe de plus en plus homogène. II faut donner aux musiciens des outils qui leur permettent de se coordonner. II faut construire des partitions qui sont des partitions surdéterminées, c'està-dire qui renferment des possibilités multiples que les musiciens peuvent utiliser à leur gré.

C'est une espèce d'expérimentation qui n'est pas vraiment un modèle ni même un exemple au sens fort des termes. C'est un champ de mise à l'épreuve qui pourrait être transposé ensuite à d'autres niveaux. Ernst Bloch utilise la notion de "mise à l'épreuve sur l'exemple» (il parle du théâtre). Je pense qu'en musique, on peut faire ce type de travail, notamment en pédagogie, en essayant d'apprendre aux jeunes musiciens à ne pas être simplement des machines qui exécutent, même avec beaucoup de conscience. Ils doivent évidemment pouvoir le faire aussi, mais ils doivent surtout être capables de créer des choses ensemble et de ne pas se détruire mutuellement. Ils doivent être capables de partager leur liberté. Voilà. C'est la grande leçon à retenir. Elle donne lieu à une sensibilité musicale d'une autre espèce, qui n'est pas la sensibilité tonale, mais plutôt la sensibilité postwebernienne, dans laquelle il peut y avoir toutes sortes de souvenirs de beaucoup d'autres musiques, à la condition que les musiciens se respectent mutuellement et qu'il n'y en ait aucun qui domine les autres.

Jean Boivin.- Ce qui m'a frappé dans vos écrits, c'est que vous parlez des propriétés de la musique, par exemple, de ses fonctions, de l'usage, des usages qu'on peut en faire, qu'on en a fait au fil des siècles, pas seulement dans la culture occidentale, mais aussi dans différentes cultures. C'est intéressant parce que vous avez une vision de l'Histoire très frappante, souvent socialisante lle mot n'est pas péjoratif dans mon esprit). Vous avez parfois des interprétations de phénomènes historiques qui sont surprenantes et qui portent à réflexion. Vous associez, par exemple, le phénomène de la monodie grégorienne du Moyen Âge au pouvoir total ecclésiastique qui dominait alors...

Henri Pousseur. - ... à l'obéissance!

Jean-Boivin.- Vous parlez aussi de l'harmonie tonale en fonction des régimes, de la hiérarchie aristocratique. Vous parlez également du rôle de la musique, de 
son action... Je voudrais ici insister sur une idée que je n'ai jamais vue ailleurs, dans d'autres textes de ce genre : vous liez l'action de la musique au concept du bonheur. Vous dites, par exemple, dans "La musique ici aujourd'hui»:

La musique, par ses différentes formes d'insertion dans la vie collective, peut certainement contribuer, fût-ce à titre de symbole, de modèle et de stimulant, à changer la société, le monde humain, I'Histoire. La musique offre à l'homme moderne l'occasion d'exercer ses facultés les plus diverses, l'acuité de ses perceptions, la pénétration de ses pensées compréhensives ou combinatoires, la vitesse, la justesse et la finesse de ses réflexes. Elle lui permet d'expérimenter des modèles de visions cosmiques, aussi bien que de réaliser des actions exemplaires pour la circulation et la coopération sociales, fêtes susceptibles de prendre des dimensions dont nous n'avons encore fait que commencer de rêver.

Toujours en fonction d'un potentiel de développement du bonheur de ceux que vous nommez les enfants de demain, vous avez certaines idées qui, pour moi, s'apparent à celles de Platon. Vous dites ceci :

[la musique] peut, entre autres, pour parler d'utilités immédiates, aider sérieusement à l'étude des langues ou de la gymnastique, à la compréhension des mathématiques ou de l'Histoire.

Ma question est utopique peutêtre, mais est-ce que vous pensez que, pour un compositeur d'aujourd'hui ou des années 1960, qui a accès à une masse d'informations musicales et culturelles sans limite (toute la musique pratiquement à travers la planète a été enregistrée), cette masse d'informations peut devenir inhibante?

Henri Pousseur.- Je vais d'abord, répondre à la question sur l'enseignement général. C'est une idée qui n'est pas de moi seul : des professeurs hongrois, par exemple, ont fait de telles expérimentations sur des classes... (en Belgique, dans l'enseignement général, la musique a totalement disparu). En France, il y a quand même un petit mouvement qui fait qu'elle revient un peu. Chez nous, les enfants qui veulent faire de la musique, même à petites doses, doivent aller dans des écoles spécialisées, en plus de l'école générale, ou alors à des cours particuliers. C'est là une grande lacune, pas seulement en ce qui concerne la musique, naturellement, mais les arts en général!

$\|$ y a donc des professeurs hongrois qui ont fait des expériences qui permettent de constater que les enfants qui ont un cours régulier de musique ont toute une série de facultés mieux aiguisées, mieux cultivées, ils sont aussi mieux éveillés. Cela leur permet de mieux suivre un certain nombre de cours. II y aurait tout un débat sur la nature même de notre enseignement qui est de plus en plus tourné vers la rentabilité rapide, etc. La technoscience prend presque tout l'espace!

Jean-Boivin.- Mais ce qui m'a frappé, entre autres, c'est que dans plusieurs discours qu'on peut lire sur la musique contemporaine depuis les années 1950 
(depuis les textes célèbres de Boulez, par exemple), il y a beaucoup d'oppositions, de prises de position contre telle et telle chose alors que, vous, vous parlez de bonheur... Je reviens donc à cette idée qui m'a frappé... La musique, pour vous, est liée à un potentiel de bonheur?

Henri Pousseur. - Oui, sûrement. Je ne sais pas si je suis un cas particulier, mais je dois à la musique une bonne partie du bonheur que j'ai éprouvé dans la vie. Ce n'est pas la seule source, loin de là li'ai des enfants, des petits-enfants, une famille et beaucoup d'autres choses, je me promène dans la nature, je jardine, je m'intéresse à d'autres arts, je lis, etc.), mais la musique, si elle est riche, ne donne pas simplement un bonheur de rêvasserie ou de transes faciles...

Jean-Boivin.- ... un bonheur passif...

Henri Pousseur. - Ça peut être une forme de bonheur riche qui inclut les facultés intellectuelles, qui rassemble les facultés de l'individu. C'est une question de culture, et l'enseignement, donc, la pédagogie sont très importants pour cela ${ }^{3} \ldots$

Mais je n'ai pas encore répondu à la fin de votre précédente question, quant à savoir si l'hypertrophie d'information dans laquelle nous baignons ne peut pas être inhibante au bout du compte.

Je voudrais y répondre d'une manière dialectique : toutes les expressions artistiques que nous rencontrons aujourd'hui, venant de tant d'époques et de tant de régions différentes, proviennent tout de même de l'espèce humaine, dont tous les sous-groupes sont interfécondables. C'est vrai non seulement sur le plan biologique, mais aussi sur celui de la culture. Toute œuvre, même de la population la plus étrangère, la plus lointaine dans le temps ou dans l'espace, la plus différente de nos propres mœurs (d'ailleurs en évolution accélérée), est chargée d'un message dont nous pouvons être les destinataires, les récepteurs, les bénéficiaires, si nous faisons preuve de modestie, et d'une écoute suffisamment ouverte (pour reprendre cette belle notion de Barthes à laquelle Isabelle Panneton a fait référence). Certes, plus nous connaîtrons le contexte, l'aurons vécu, éprouvé ou reconstituté, plus notre compréhension sera pénétrante. Mais même la pièce isolée dans un musée, l'enregistrement transplanté sans autre commentaire, conservent dans les meilleurs des cas une puissance d'information qui peut être pour nous une grande source de transformation, d'enrichissement (immatériel!) et d'amélioration.

Comparer plusieurs de ces émetteurs de haute tension, les mettre en contact, les composer et les faire jover ensemble (surtout selon le principe webernien) aura une portée encore plus générale, faisant apparaître l'universel commun sans pour autant perdre les différents particuliers.

Ça, c'est dans le cas d'œuvres, de messages de très haute tenue, de très forte charge.

Mais nous assistons certes aujourd'hui, non seulement du fait des médias et de la recherche effrénée du profit qui les anime, mais aussi lles deux sont "en che-
3. Les paragraphes qui suivent et qui complètent sa réponse ont été écrits par Henri Pousseur en juin 2001 pour remédier à une malheureuse interruption technique nous ayant privé de l'enregistrement de la fin de cette table ronde de janvier 2001 
ville», se conditionnent mutuellement) de la frénésie de consommation qui recherche constamment la nouveauté, fût-elle factice, et qui anime surtout les couches les plus désenchantées que cette société n'arrête pas de produire len particulier parmi la jeunesse plus ou moins révoltée - avec raisons!|, nous assistons à un "métissage " qui va tellement loin qu'il aboutit à une sorte de concassage, de réduction en presque poussière (philosophiquement digne d'être méditée!), ou alors de régression vers l'ultra-élémentaire; aucun de ces phénomènes ne devant, à mon sens, être méprisé et balayé (en esprit, car "dans le réel», cela se défend bec et ongles!) du revers de la main.

Car n'est-ce pas peut-être, au bout de nos culbutes, de nos tourbillons et de notre tourmente, la production d'une sorte de terreau sur lequel (ou dans lequel) on peut espérer que des semences non détruites finiront par germer, et par produire les nouveaux organismes dans lesquels l'avenir pourra vivablement s'incarner?

Quant à savoir si nous contribuons à la tourbe ou à la préservation de quelques graines, les paris sont ouverts. 